\title{
Inaccuracies in the Use of Magnification Markers in Digital Hip Radiographs
}

\author{
Michael J. Archibeck MD, Tamara Cummins RT, Krishna R. Tripuraneni MD, \\ Joshua T. Carothers MD, Cristina Murray-Krezan MS, Mohammad Hattab PhD, \\ Richard E. White Jr MD
}

Received: 21 October 2015/Accepted: 14 December 2015/Published online: 21 January 2016

(C) The Association of Bone and Joint Surgeons \& 2016

\begin{abstract}
Background With the ubiquity of digital radiographs, the use of digital templating for arthroplasty has become commonplace. Although improved accuracy with digital radiographs and magnification markers is assumed, it has not been shown.
\end{abstract}

One of the authors certifies that he (KRT) or a member of his immediate family, has or may receive payments or benefits, during the study period, an amount of less than USD 10,000 from Orthoview Software (Jacksonville, FL, USA).

All ICMJE Conflict of Interest Forms for authors and Clinical Orthopaedics and Related Research ${ }^{\circledR}$ editors and board members are on file with the publication and can be viewed on request.

Clinical Orthopaedics and Related Research ${ }^{\mathbb{R}}$ neither advocates nor endorses the use of any treatment, drug, or device. Readers are encouraged to always seek additional information, including FDAapproval status, of any drug or device prior to clinical use. Each author certifies that the Presbyterian Hospital, Albuguerque, $\mathrm{NM}$, approved or waived approval for the human protocol for this investigation and that all investigations were conducted in conformity with ethical principles of research.

M. J. Archibeck, T. Cummins, K. R. Tripuraneni, J. T. Carothers, R. E. White Jr

New Mexico Orthopaedics, Albuquerque, NM, USA

\section{Murray-Krezan}

Division of Epidemiology, Biostatistics, and Preventive

Medicine, Department of Internal Medicine, University of New

Mexico Health Sciences Center, Albuquerque, NM, USA

\section{Hattab}

Department of Math and Statistics, University of New Mexico, Albuquerque, NM, USA

M. J. Archibeck ( $\square)$

New Mexico Orthopaedics, 201 Cedar SE, Suite 6600,

Albuquerque, NM 87106, USA

e-mail: archibeckmj@nmortho.net
Questions/Purposes We wanted to (1) evaluate the accuracy of magnification markers in estimating the magnification of the true hip and (2) determine if the use of magnification markers improves on older techniques of assuming a magnification of $20 \%$ for all patients.

Methods Between April 2013 and September 2013 we collected 100 AP pelvis radiographs of patients who had a THA prosthesis in situ and a magnification marker placed per the manufacturer's instructions. Radiographs seen during our standard radiographic review process, which met our inclusion criteria (AP pelvic view that included a well-positioned and observed magnification marker, and a prior total hip replacement with a known femoral head size), were included in the analysis. We then used OrthoView ${ }^{\mathrm{TM}}$ software program to calculate magnification of the radiograph using the magnification marker (measured magnification) and the femoral head of known size (true magnification).

Results The mean true magnification using the femoral head was $21 \%$ (SD, 2\%). The mean magnification using the marker was $15 \%$ (SD, 5\%). The 95\% CI for the mean difference between the two measurements was $6 \%$ to $7 \%$ (p $<0.001)$. The use of a magnification marker to estimate magnification at the level of the hip using standard radiographic techniques was shown in this study to routinely underestimate the magnification of the radiograph using an arthroplasty femoral head of known diameter as the reference. If we assume a magnification of $20 \%$, this more closely approximated the true magnification routinely. With this assumption, we were within $2 \%$ magnification in 64 of the 100 hips and off by $4 \%$ or more in only four hips. In contrast, using the magnification marker we were within $2 \%$ of true magnification in only 20 hips and were off by $4 \%$ or more in 59 hips.

Conclusion We found the use of a magnification marker with digital radiographs for preoperative templating to be 
generally inaccurate, with a mean error of $6 \%$ and range from $-5 \%$ to $15 \%$. Additionally, these data suggest that the use of a magnification marker while taking preoperative radiographs of the hip may be unnecessary, as simply setting the software to assume a $20 \%$ magnification actually was more accurate.

Level of Evidence Level III, diagnostic study.

\section{Introduction}

The value of preoperative templating has been described for THA [3]. Benefits include estimation of appropriate acetabular and femoral component sizing, appropriate restoration of preoperative offset, correction of leg length discrepancy, and anticipation of special componentry or techniques. Before introduction of digital radiographs, templating typically involved the use of analog radiographs and acetate onlay templates. These onlay templates typically assumed a magnification of $20 \%$. With the introduction of digital radiographs, templating techniques have evolved. Generally, digital preoperative radiographs are performed with a magnification marker. The templating software then allows the user to measure the magnification and the templates are scaled to the appropriate magnification. The use of a magnification marker at the time of radiograph acquisition adds time and expense to the process. Thus far, there is no evidence that it adds accuracy.

The accuracy of digital templating relies on the accuracy of the magnification marker and its ability to estimate true magnification of the bone at the level of the hip. Placement of the marker too close or far from the radiographic plate relative to the coronal plane of the hip will result in underestimation or overestimation of the magnification respectively. With this in mind, Sinclair et al. [4] recently reported their results of a study comparing the actual magnification of the hip by using the femoral head of a previously placed hip arthroplasty prosthesis with the magnification of the magnification marker. They found a mean discrepancy between the two measurements of 7\% (range, 0\%-26\%), concluding that such errors may result in incorrect component sizes, leg lengths, and offset [4]. To date, there has been no publication, to our knowledge, showing superiority of the magnification marker or a comparison of a standard magnification with the use of the marker.

We therefore asked two clinical questions: (1) Does the use of externally placed magnification markers on preoperative hip radiographs provide an accurate measurement of the true magnification at the level of the hip? (2) Is the use of digital radiography and magnification markers in preoperative templating for THA an improvement over the prior standard $20 \%$ magnification "onlay" techniques?

\section{Methods}

All preoperative digital radiographs in our facility are taken with a magnification marker placed between the legs of the patient on the AP pelvis for templating. We sought to identify such AP pelvis radiographs that were obtained on patients with a prior contralateral total hip replacement with a known femoral head size. Between April and September 2013, we collected 100 AP pelvis radiographs from patients with a prior THA prosthesis in situ and a magnification marker placed per the manufacturer's instructions (Fig. 1). These radiographs were collected for this study as they were encountered during our routine radiographic followup analysis. As our research radiology reviewer (TC) identified images that met the inclusion criteria (a high-quality AP pelvis radiograph with a wellpositioned magnification marker, and a previously performed total hip replacement), she enrolled the images in the study. These 100 images were identified among approximately 1200 that were reviewed. These patients' THAs had been performed by one of the surgeons at our facility and, thus, the femoral head size and material were known to us. We excluded any THAs performed with an enhanced ingrowth surface (Trabecular Metal ${ }^{\mathrm{TM}}$; Zimmer, Warsaw, IN, USA) as the contour of the femoral head was difficult to detect. The magnification marker (J2 Medical LP, Pittsburgh, PA, USA) is a $25-\mathrm{mm}$ metallic sphere that is snapped into a flexible arm that attaches to the table using a suction cup. The greater trochanter is used to determine the level of bone, or desired coronal plane. The flexible sphere is placed next to the patient and adjusted to that level. The device then is moved and placed between the legs. It was placed by trained radiology technicians per the instructions of the manufacturer. We then used

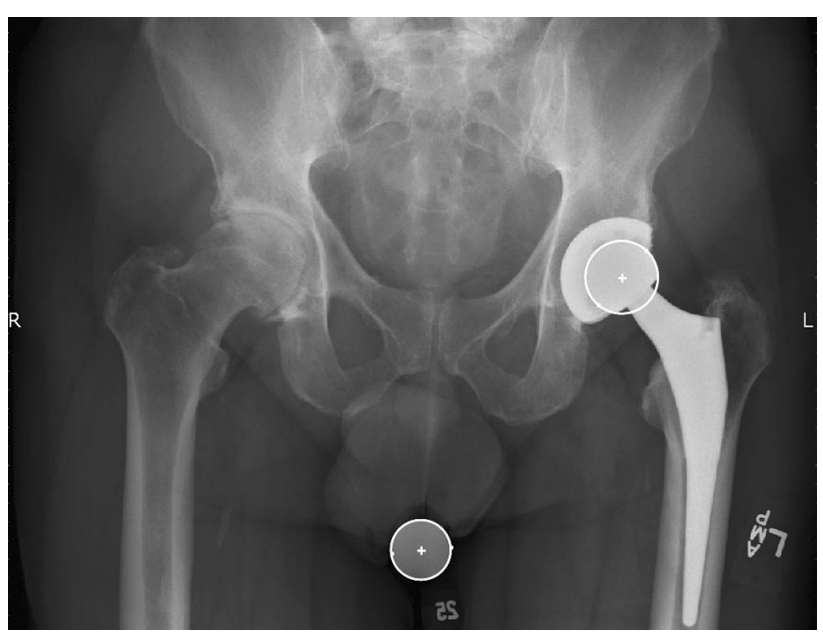

Fig. 1 An AP pelvic radiograph is shown with a central magnification marker and THA that was used for measuring "measured" and "true" magnification respectively. 
OrthoView $^{\text {TM }}$ software (Jacksonville, FL, USA) to calculate magnification of the radiograph using the magnification marker and the femoral head of known size. This was performed by tagging the femoral head and the magnification marker and adjusting the size of the circle to conform to the contour of the femoral head. The femoral head is a truncated sphere such that the portion of the head that fits onto the stem is flattened. The sphere is placed on the head to best fit the contour of the spherical portion of the head. We also collected the demographics of the patients including the BMIs.

All AP pelvic radiographs were obtained with the patient in a supine position and were considered to be of high quality with excellent visualization and symmetry of the pelvis and the entirety of both hips and proximal femurs. The magnification marker was well observed and adjacent to the soft tissues centrally. The x-ray tube was centered over the symphysis pubis and placed 40 inches above the patient. The images were obtained in one of five identical radiography examination rooms in one clinic with multiple radiographers. All of the images were digital and were obtained using Quantum Medical imaging equipment (Quantum Medical, LLC, Ronkonkoma, NY, USA). They all had Canon high sensitivity digital detectors (Canon USA, Long Island, NY, USA). A 40-inch distance from the pubis is the standard protocol for this facility. The $25-\mathrm{mm}$ magnification marker was fully visible on the image. The head size was documented by the prior operative reports.

We considered the magnification measured using the THA femoral head of known size to be the "true magnification". The magnification determined using the $25-\mathrm{mm}$ magnification marker was acquired and labeled the "measured magnification". The radiographic review was performed by a radiology technician and clinical researcher (TC) with years of experience in radiographic evaluation of hip arthroplasties.

\section{Statistical Analysis}

An intraobserver reliability study was conducted of the radiology technician (TC) who performed all measurements. First, we performed a power analysis that indicated we needed to conduct three measurement trials on 25 patients to have $95 \%$ power to detect an intraclass correlation coefficient (ICC) of at least 0.80 . A random sample of 25 study identification numbers (IDs) was selected from the 100 samples collected. Next, we randomized the order in which the IDs were to be read and whether the first read should be a "true" magnification or a "measured" magnification for each of three independent trials. We fitted a linear mixed model with variance components covariance structure to the individual measurements with independent variables of trial number, type of measurement ("true" or "measured"), and an interaction between the two, along with a random intercept for study ID to account for the repeated measures on the 25 subjects. Least-squares means and $95 \%$ CIs were calculated from the model for the each type of measurement. The ICC of the differences was calculated from the variance components from repeated measures ANOVA models for individual measurement types and the differences between types using standard methods [5].

For the intraobserver reliability study, the linear mixed model fitted to the magnification measurements indicated no interaction effect between trial and type of magnification (true or measured; $\mathrm{F}=0.04, \mathrm{p}=0.957$ ). A reduced model with just independent variables of trial number and type of magnification revealed that only type affected the measurement size $(F=113.04, p<0.001)$. For the reliability study, the adjusted mean (least-squares) estimate of the true magnification was $21 \%$ (95\% CI, 20\%- 23\%) whereas the measured magnification estimate was $15 \%$ (95\% CI, 14\%-16\%). Finally, the calculated ICC for the repeated trials of the paired differences was 0.93 . Because trial number was not statistically significant in the analyses and the ICC was quite high, we concluded that we had consistent intraobserver reliability.

The primary aim of the study was to determine the accuracy of using the magnification marker for preoperative templating compared with the true magnification. A power analysis based on the mean percent difference between measurement types $(\Delta)$ indicated that a sample size of 100 pairs was sufficient to produce a two-sided $95 \%$ CI with width $\Delta \pm 1.0 \%$ assuming an estimated SD of the paired differences of $5.0 \%$. A simple linear regression model was fitted to the paired differences in measurement types with the single independent variable BMI to assess the relationship between the differences and BMI. To estimate the mean and size of the discrepancy, a multiple linear regression model was fitted to the measurement values with independent variables including BMI, type of measurement (true or measured), and an interaction between the two. The least-squares mean values and their 95\% CIs were calculated from the regression model. Normality of the residuals was assessed. We also created a Bland-Altman plot to visually compare the true and measured values. In such a plot, the mean of the two types of measurements are displayed on the horizontal axis and the difference between them on the vertical axis. The 95\% limits of agreement were overlaid. Typically, good agreement means that the points in this plot are spread randomly about the mean difference between the two types of measurement with most points falling between the $95 \%$ limits of agreement. Probability values were considered statistically significant if they were less than the type I error rate 
of $\alpha=0.05$. Analysis was performed using SAS ${ }^{\circledR} 9.4$ (SAS Institute Inc, Cary, NC, USA) and the graph was made in Stata $14 / \mathrm{IC}^{\mathrm{TM}}$ (StataCorp, College Station, TX, USA).

\section{Results}

We found that the use of a magnification marker did not accurately predict the actual magnification of the bone at the level of the hip as desired for preoperative templating. The true magnification (determined by using the femoral head of the THA prosthesis in situ) ranged from $17 \%$ to $27 \%$ with a mean of $21 \%$ (SD, $2 \%$ ). Measured magnification (using the placed magnification marker) ranged from $7 \%$ to $30 \%$ with a mean of $15 \%$ (SD, $5 \%$ ). True magnification measurements were larger than the measured by a mean of 6\% (SD, 5\%; p < 0.001) (Table 1). Simple linear regression of the paired differences between measurement types and BMI showed that BMI did not affect the paired differences $(F=1.38, p=0.243)$. However, when the multiple regression model was fitted to the measurement value, the interaction between BMI and type of measurement (true vs measured) was not significant, but both main effects were (BMI: $\mathrm{F}=22.84, \mathrm{p}<0.001$; Type: $\mathrm{F}=129.77$, $\mathrm{p}<0.001)$. This means that BMI accounted for a significant amount of variability in the measurement sizes; however, there was not a differential effect of BMI on the two types of measurements. True values were consistently larger than measured values with an adjusted mean (leastsquares) difference of $6 \%$ (95\% CI, 5\%-7\%). The residuals in all linear models were approximately normally distributed indicating good model fitting. A Bland-Altman plot of the types of measurements is shown (Fig. 2). Most values fell within the $95 \%$ limits of agreement around the mean difference of 6\%; however, a distinct negatively decreasing pattern was evident indicating that as the measurements increased in diameter, the difference between the true and measured values became smaller.

In addition, the use of a magnification marker with digital hip radiographs did not provide a more-accurate measurement of hip magnification than the use of a standard 20\% magnification for all patients. Comparison of each hip showed a discrepancy between the true and measured magnification ranging from $-5 \%$ to $15 \%$ (Table 1). Eighty-eight percent ( 88 of 100 hips) of the magnification markers underestimated the true magnification (marker placed too close to the table) by a mean of $7 \%$ (range, 1\%-15\%). In seven hips the magnification marker overestimated the magnification by a mean of $4 \%$ (range, $1 \%-5 \%)$. In five hips, the magnification of the marker was the same as the true magnification. Because the true magnification was very close to the standard $20 \%$ magnification assumed on older acetate onlay templates and had a relatively small SD, we descriptively compared the "old technique" of assuming a $20 \%$ magnification with the digital technique of using the magnification marker to scale the template sizes (Table 2). If we assumed that all radiographs were $20 \%$ magnified, we were within $2 \%$ magnification in 64 of the 100 hips and off by $4 \%$ or greater in only four hips. In contrast, using the magnification marker to scale the templates, we were within $2 \%$ magnification in only 20 of the 100 hips and were off by $4 \%$ or more in 59 hips. We chose $4 \%$ as this likely

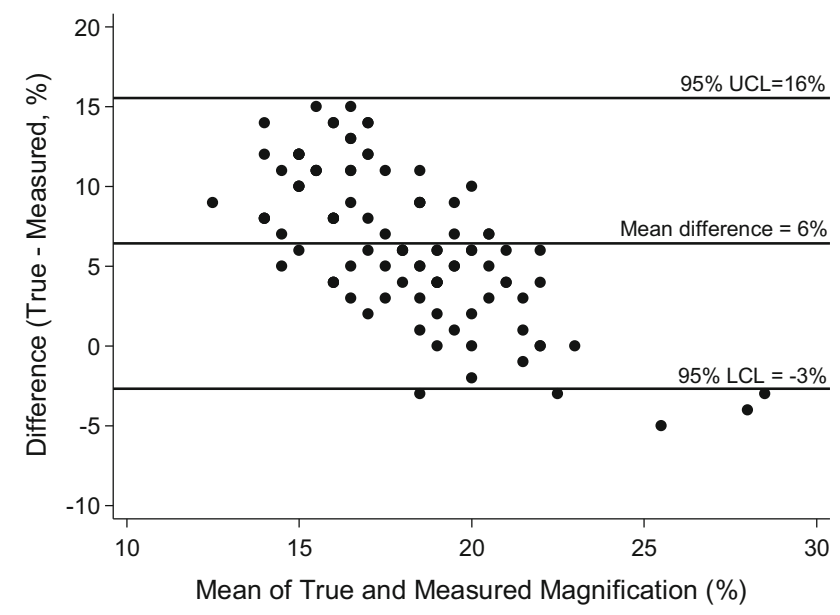

Fig. 2 The Bland-Altman plot shows the difference between the true and measured magnification values versus the mean of the two magnification types and the $95 \%$ limits of agreement (UCL $=$ upper confidence limit, $\mathrm{LCL}=$ lower confidence limit). As the measurement values increased, the difference between the two types of measurements became smaller.

Table 1. Comparison of magnification method results

\begin{tabular}{lllll}
\hline $\begin{array}{l}\text { Statistical } \\
\text { parameter }\end{array}$ & $\begin{array}{l}\text { True percent magnification } \\
\text { (THA prosthesis head) }\end{array}$ & $\begin{array}{l}\text { Measured percent } \\
\text { magnification (marker) }\end{array}$ & $\begin{array}{l}\text { Difference between measurement } \\
\text { types (true-measured) }\end{array}$ & $\begin{array}{l}\text { p value for } \\
\text { difference* }\end{array}$ \\
\hline Number & 100 & 100 & 100 & NA \\
Mean \pm SD & $21 \pm 2$ & $15 \pm 5$ & $6 \pm 5$ & $<0.001$ \\
$95 \% \mathrm{CI}^{\dagger}$ & $(21-22)$ & $(14-16)$ & $(6-7)$ & NA \\
Range & $(17-27)$ & $(7-30)$ & $(-5$ to 15) & NA \\
\hline
\end{tabular}

* $\mathrm{p}$ value obtained from $\mathrm{t}$ test of least-squares mean estimates of magnification types adjusted for BMI; ${ }^{\dagger}$ confidence interval for least-squares mean estimates of percent magnification adjusted for BMI; NA = not applicable. 
Table 2. Comparison of radiograph measurements using the magnification marker versus standard $20 \%$ magnification

\begin{tabular}{lll}
\hline Measurement method & $\begin{array}{l}\text { Using } \\
\text { magnification } \\
\text { marker }\end{array}$ & $\begin{array}{l}\text { Using a standard } \\
20 \% \\
\text { magnification* }\end{array}$ \\
\hline $\begin{array}{l}\text { Measurement within 2\% of "true } \\
\text { magnification" }\end{array}$ & $20 / 100$ & $64 / 100$ \\
$\begin{array}{l}\text { Measurement greater than 4\% } \\
\text { deviation from "true } \\
\text { magnification" }\end{array}$ & $59 / 100$ & $4 / 100$ \\
\hline
\end{tabular}

* Standard $20 \%$ magnification assumed on older acetate onlay templates.

represents at least one size of femoral or acetabular component.

\section{Discussion}

The value of preoperative templating before a THA has been well established [6-8]. Accurate preoperative templating depends on many factors including the ability to measure or estimate the magnification of the radiographic images at the level of the hip. Before routine use of digital radiographs, onlay templating typically assumed a magnification of $20 \%$. With digital radiographs, the use of a standard-sized magnification marker, typically $25 \mathrm{~mm}$ in diameter, placed at the estimated level of the bone by the radiology technician has become commonplace, with no proof of increased accuracy compared with older techniques. The use of the magnification marker adds additional cost and time to the process. This study was designed to address two clinical questions: (1) Does the use of externally placed magnification markers on preoperative hip radiographs provide an accurate measurement of the true magnification at the level of the hip? (2) Is the use of digital radiography and magnification markers in preoperative templating for THA an improvement over the prior standard 20\% magnification "onlay" techniques?

There are several weaknesses of this study. The isolated use of the AP pelvis view may not be an accurate representation of templating using the AP view of the hip. The common errors in placement of the magnification marker may be unique to our facility and training biases. We looked at the ability to measure magnification and not the ability to actually predict implanted componentry. Additionally, the variable radiology technicians who took the radiographs lends a potential source of error. This may lead to an exaggeration in the differences in magnification if not done meticulously. All radiology technicians have been trained in using the magnification marker as per the manufacturer's instructions. Based on the results of this study, we have informed them of the common errors and reinforced the technique. However, this is a real-to-life assessment of the many radiology technicians taking radiographs in most practices. Additionally, the use of manually adjusted circles about a radiographic sphere lends itself to potential error. However, this is the actual technique as developed and used by a majority of surgeons. This is the message of the study: the use of a magnification marker has many potential sources of error, and perhaps it should be abandoned and replaced with a more-accurate standard $20 \%$ magnification. We also did not quantify the coronal plane locations of the femoral head center and the magnification marker as this difference can affect relative magnification. Additionally, we were unable to quantify the effect of BMI on these measurements among patients. However, BMI did not affect the conclusion that the magnification marker did not provide additional accuracy compared with a standard magnification. We also did not study the clinical implications of these errors in magnification with respect to component sizing, leg length, or offset. Although our study is not intended to show detrimental effect on the component size selection or geometry of the reconstruction, it is reasonable to suggest that more accuracy is better. In this case, the less-expensive technique of setting the software to a standard $20 \%$ magnification is more accurate compared with the more cumbersome and expensive use of the magnification marker.

We found a mean error using the magnification marker of $6 \%$ with a range from $-5 \%$ to $15 \%$. The marker underestimated the magnification in $88 \mathrm{hips}$, indicating the common error in radiology technician placement of the marker ball to be too close to the table. In addition to erroneous measurement of the magnification using the marker, the range of measurements was quite large (range, $7 \%-30 \%)$. Little has been published in this area [2, 7]. In their Instructional Course Lecture, Whiddon and Bono [7] reported on the technique of digital templating. They commented that acetate onlay templating is subject to errors in magnification and thought the use of magnification markers would allow increased accuracy. Iorio et al. [2] compared the accuracy of acetate and digital templating in its ability to predict the implants used in total hip replacement in 50 hips and found the absolute errors to be greater in digital templating, but not the difference in mean errors. They concluded that digital templating is acceptably safe for preoperative planning [2]. Since then, several studies have investigated the ability of digital templating to predict the implanted componentry at the time of arthroplasty [1, 6, 8]. Gamble et al. [1] found that digital templating showed good intraobserver and interobserver reliability. Digital templating predicted to within one size of the femoral component in $85 \%$ of hips and $80 \%$ of the 
acetabular components. Acetate templating was accurate to within one size in $85 \%$ of the femoral components and $60 \%$ of acetabular components. Similarly, Steinberg et al. [6] studied 73 hips retrospectively and found digital templating to predict to within one size in $89 \%$ of acetabular components and $97 \%$ of femoral components. Whiddon et al. [8] found digital templating predictive to within one size in $78 \%$ of acetabular components and $90 \%$ of femoral components. None of these investigations actually assessed the accuracy of the magnification calculations. Similar to our study, Sinclair et al. [4] recently compared the actual magnification of the hip by using the femoral head of a previously placed hip arthroplasty prosthesis with the magnification of the magnification marker. They found a mean error between the two measurements of $7 \%$ (range, $0 \%-26 \%$ ), concluding that such errors may result in incorrect component sizes, leg lengths, and offset [4].

We found the true magnification mean of $21 \%$ to be consistent with prior onlay templating estimates of $20 \%$, and with a smaller range (range, 17\%-27\%). This suggests that assuming a magnification of $20 \%$ is more accurate than the use of the magnification marker. The actual difference in mean magnification was modest at $6 \%$ and clinically may represent only one to two component sizes. However, using a standard $20 \%$ magnification was shown to approximate the actual magnification more accurately, had a smaller deviation, and avoided the occasional aberrant results of the magnification marker. These results suggest that, even with the use of digital radiographs, it may be more accurate to simply set the software magnification to $20 \%$ or $21 \%$ than use the magnification marker, thus avoiding the added expense and time needed to use the marker.

Our study showed that the use of magnification markers at the time of digital radiograph acquisition for preoperative hip imaging does not accurately estimate the actual magnification at the level of the hip. Additionally, the use of a standard magnification value, such as $20 \%$, actually provides improved accuracy and avoids the added expense and time involved with the use of such magnification markers. Surgeons should be aware of the inaccuracies associated with the use of digital radiographs and magnification markers. Finally, these data should cause us to ask whether magnification markers are necessary or even helpful, given the reduced error associated with using an assumed magnification of $20 \%$ in this series.

\section{References}

1. Gamble P, de Beer J, Petruccelli D, Winemaker M. The accuracy of digital templating in uncemented total hip arthroplasty. $J$ Arthroplasty. 2010;25:529-532

2. Iorio R, Siegel J, Specht LM, Tilzey JF, Hartman A, Healy WL. A comparison of acetate vs digital templating for preoperative planning of total hip arthroplasty: is digital templating accurate and safe? J Arthroplasty. 2009;24:175-179.

3. Shaarani SR, McHugh G, Collins DA. Accuracy of digital preoperative templating in 100 consecutive uncemented total hip arthroplasties: a single surgeon series. $J$ Arthroplasty. 2013;28:331-337.

4. Sinclair VF, Wilson J, Jain NP, Knowles D. Assessment of accuracy of marker ball placement in pre-operative templating for total hip arthroplasty. J Arthroplasty. 2014;29:1658-1660.

5. Stanish WM, Taylor N. Estimation of the intraclass correlation coefficient for the analysis of covariance model. Am Stat. 1983; 37:221-224.

6. Steinberg EL, Shasha N, Menahem A, Dekel S. Preoperative planning of total hip replacement using the TraumaCad ${ }^{\mathrm{TM}}$ system. Arch Orthop Trauma Surg. 2010;130:1429-1432.

7. Whiddon DR, Bono JV. Digital templating in total hip arthroplasty. Instr Course Lect. 2008;57:273-279.

8. Whiddon DR, Bono JV, Lang JE, Smith EL, Salyapongse AK. Accuracy of digital templating in total hip arthroplasty. Am J Orthop (Belle Mead NJ). 2011;40:395-398. 